\title{
$\rho$ meson GPDs and IPDs with a light-front constituent quark model
}

\author{
Bao-Dong Sun ${ }^{1,2, *}$ and Yu-Bing Dong ${ }^{1,2,3}$ \\ ${ }^{1}$ Institute of High Energy Physics, Chinese Academy of Sciences, Beijing 100049, China \\ ${ }^{2}$ School of Physics, University of Chinese Academy of Sciences, Beijing 100049, China \\ ${ }^{3}$ Theoretical Physics Center for Science Facilities (TPCSF), CAS, Beijing 100049, China
}

Abstract. We investigate the $\rho$ meson unpolarized Generalized Parton Distributions based on a Light-Front Constituent Quark Model. Moreover, by employing a Gaussian form wave packet, we apply the obtained generalized parton distributions to get the Impact Parameter dependent parton Distributions.

\section{Introduction}

It is well-known that the usual parton distributions (PDFs) can give the longitudinal information of hadron targets in the deep inelastic scattering (DIS) processes. To investigate the transverse information, the generalized parton distributions (GPDs) could be a powerful tool [1]. In our recent work, a Light-Front Constituent Quark Model (LFCQM) is employed to study the GPDs [2] of the $\rho$ meson (spin-1) and its Impact Parameter dependent parton Distributions (IPDs) [3].

\section{GPD and our model}

The unpolarized GPDs of a spin-1 particle $H_{i}, i=1,5$ are defined as [4]

$$
V_{\lambda^{\prime} \lambda}=\left.\frac{1}{2} \int \frac{d \omega}{2 \pi} e^{i x(P z)}\left\langle p^{\prime}, \lambda^{\prime}\left|\bar{q}\left(-\frac{1}{2} z\right) h q\left(\frac{1}{2} z\right)\right| p, \lambda\right\rangle\right|_{z=\omega n}=\sum_{i} \epsilon^{\prime * v} V_{\nu \mu}^{(i)} \epsilon^{\mu} H_{i}^{q}(x, \xi, t)
$$

where $\epsilon$ (or $\epsilon^{\prime}$ ) is the polarization vector of the incoming (or outgoing) spin-1 particle, and $V_{v \mu}^{(i)}$ are Lorentz structures. By taking the forward limit, one can obtain the $\rho$ meson PDF through GPD $H_{1}$ as $q(x)=\left[q^{1}(x)+q^{-1}(x)+q^{0}(x)\right] / 3=H_{1}^{q}(x, 0,0)$. The $\rho$ meson form factors (FFs) are related to its GPDs by the following sum-rules,

$$
\int_{-1}^{1} d x H_{i}^{q}(x, \xi, t)=G_{i}^{q}(t)(i=1,2,3), \int_{-1}^{1} d x H_{i}^{q}(x, \xi, t)=0(i=4,5),
$$

and the charge, magnetic and quadrupole form factors are given by [5]

$$
G_{C}(t)=G_{1}(t)+\frac{2}{3} \eta G_{Q}(t), G_{M}(t)=G_{2}(t), G_{Q}(t)=G_{1}(t)-G_{2}(t)+(1+\eta) G_{3}(t)
$$

The normalization condition is $G_{C}(0)=1$.

\footnotetext{
*e-mail: sunbd@ihep.ac.cn
} 
In analogy to the chiral interaction Lagrangian for the $\pi \rightarrow q \bar{q}$ vertex [6], the effective Lagrangian for the $\rho \rightarrow \bar{q} q$ is taken as

$$
\mathcal{L}_{I}=-\frac{\imath M}{f_{\rho}} \bar{q} \Gamma^{\mu} \tau q \cdot \rho_{\mu}=-\frac{\imath \sqrt{2} M}{f_{\rho}}\left[\frac{\bar{u} \Gamma^{\mu} u-\bar{d} \Gamma^{\mu} d}{\sqrt{2}} \rho_{\mu}^{0}+\bar{u} \Gamma^{\mu} d \rho_{\mu}^{+}+\bar{d} \Gamma^{\mu} u \rho_{\mu}^{-}\right],
$$

where $\rho_{\mu}$ is the massive spin one field, $f_{\rho}$ is the $\rho$ decay constant, and $\Gamma^{\mu}$ is a Bethe-Salpeter amplitude (BSA),

$$
\Gamma^{\mu}=N \frac{\gamma^{\mu}-\left(k_{q}-k_{\bar{q}}\right)^{\mu} /\left(M_{\mathrm{inv}}+2 m_{q}\right)}{\left[k_{q}^{2}-m_{R}^{2}+\imath \epsilon\right]\left[k_{\bar{q}}^{2}-m_{R}^{2}+\imath \epsilon\right]},
$$

where $N$ is the normalization constant, $m_{q}$ and $m_{R}$ are the constituent quark and the regulator masses, respectively. The bound state invariant mass $M_{\mathrm{inv}}$ is defined differently in the valence and nonvalence regions [2]. In our approach, we take $m_{q}=0.403 \mathrm{GeV}$ and $m_{R}=1.61 \mathrm{GeV}$. Our $\rho$ meson mean square charge radius $\left\langle r^{2}\right\rangle=0.52 \mathrm{fm}^{2}$, magnetic moment $\mu=2.06 /\left(2 m_{\rho}\right)$ and quadrupole moment $Q_{\rho} / m_{\rho}^{2}=-0.323$. All the calculated observables agree with experiment data and most of previous calculations [2].

The longitudinal information can be investigated when the skewness variable $\xi$ is nonzero. Our results for $\rho$ FFs and GPDs with $\xi=-0.4$ are show in Fig. 1. After leading order DGLAP evolution to Lattice scale, we find the Mellin moments of the obtained GPDs, see in Table 1, match well between our model and the Lattice QCD calculation [8].

Table 1. Mellin moments of GPDs.

\begin{tabular}{lllllll}
\hline & $\int d x x H_{1}$ & $\int d x x^{2} H_{1}$ & $\int d x x^{3} H_{1}$ & $\int d x x H_{5}$ & $\int d x x^{2} H_{5}$ & $\int d x x^{3} H_{5}$ \\
\hline Lattice QCD & $0.33(2)$ & $0.17(5)$ & $0.06(4)$ & $0.29_{-23}^{+22}$ & $-0.001(15)$ & $-0.01(6)$ \\
\hline Our result & $0.34(2)$ & $0.15(1)$ & $0.08(1)$ & $0.044(3)$ & $0.048(5)$ & $0.039(5)$ \\
\hline
\end{tabular}

\section{IPDs}

To obtain the information how PDF are distributed in position space, one can take Fourier transform of $V_{\lambda \lambda^{\prime}}$ w.r.t. the transverse momenta $\mathbf{p}_{\perp}$ and $\mathbf{p}_{\perp}^{\prime}$, which gives the so-called IPDs [3]. Experimentally there is a largest measured value $|t|_{\max }$ and thus the measurement accuracy is $\sigma \sim\left(|t|_{\max }\right)^{-1 / 2}$. To account this limit, we introduce a Gaussian form wave packet in initial and final states,

$$
G\left(\mathbf{p}_{\perp}, 1 / \sigma^{2}\right)=\exp \left(-\mathbf{p}_{\perp}^{2} \sigma^{2} / 2\right) .
$$

The IPD for $H_{1}^{q}$ (which relates to DIS structure function $F_{1}$ ) is

$$
q_{\sigma}(x, b)=\int_{0}^{\infty} \frac{\Delta_{\perp} d \Delta_{\perp}}{2 \pi} J_{0}\left(b \Delta_{\perp}\right) e^{-\Delta_{\perp}^{2} \sigma^{2} / 4} H_{1}\left(x, 0,-\Delta_{\perp}^{2}\right),
$$

with $q_{\sigma}(b)=\int_{0}^{1} d x q_{\sigma}(x, b)$.

Our results for IPDs are shown in Fig. 2. We see that as $\sigma$ becomes smaller, the wave functions of the initial and final states get closer to a plane wave, and the parton distribution becomes more transversely localized in the position space. Following the same way, IPDs for $G_{C, M, Q}$ are also introduced in our calculation.

This work is supported by the National Natural Science Foundation of China under Grant No. 11475192, by the fund provided to the Sino-German CRC 110 "Symmetries and the Emergence of Structure in QCD" project by the NSFC under Grant No.11621131001, and the Key Research Program of Frontier Sciences, CAS, Grant No. Y7292610K1. 


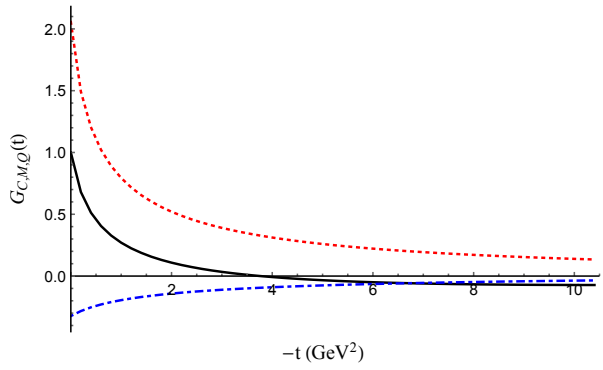

(a) $\rho$ form factors, $G_{C}$ (solid black line), $G_{M}$ (dashed red line) and $G_{Q}$ (dot-dashed blue line)

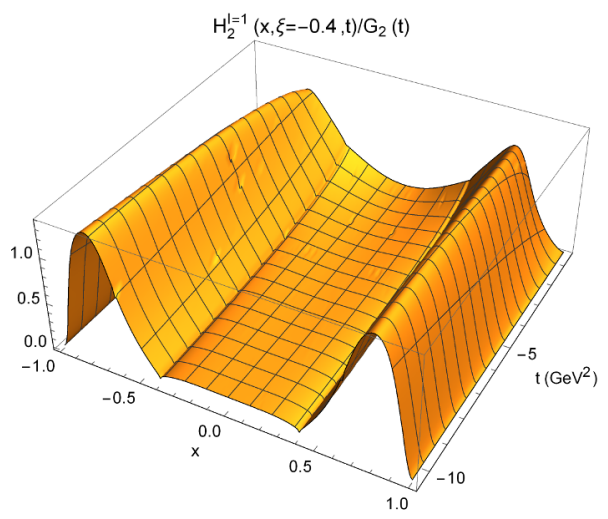

(c) $\rho$ GPD $\mathrm{H}_{2}$

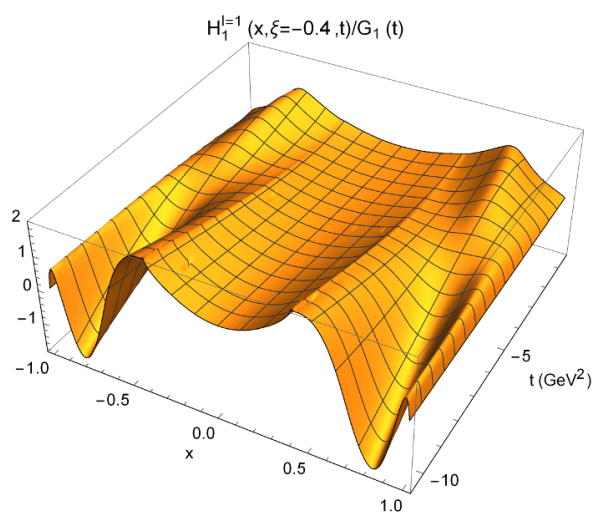

(b) $\rho$ GPD $H_{1}$

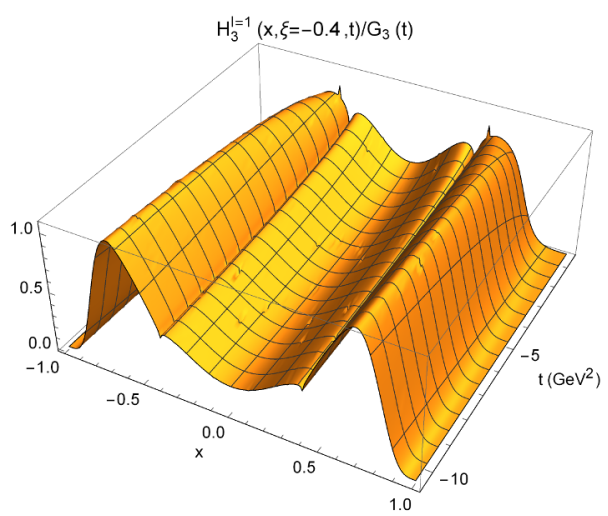

(d) $\rho$ GPD $H_{3}$

Figure 1. $\rho$ form factors and GPDs with $\xi=-0.4$.

\section{References}

[1] M. Diehl, Phys. Rept. 388, 41 (2003)

[2] B.D. Sun et al., Phys. Rev. D 96, 036019 (2017)

[3] B.D. Sun et al., Chin. Phys. C 42, 063104 (2018)

[4] E. R. Berger et al., Phys. Rev. Lett. 87, 142302 (2001)

[5] M. Garcon et al., Adv. Nucl. Phys. 26, 293 (2001)

[6] T. Frederico et al., Phys.Rev. D 45, 4207 (1992)

[7] M. Burkardt, Int. J. Mod. Phys. A18, 173 (2003)

[8] C. Best, et al., Phys. Rev. D 56, 2743(1997) 


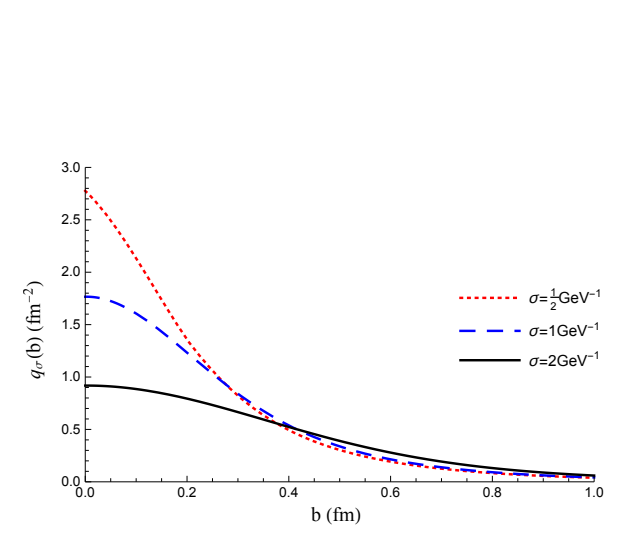

(a) $q_{\sigma}(b)\left(\mathrm{fm}^{-2}\right)$.

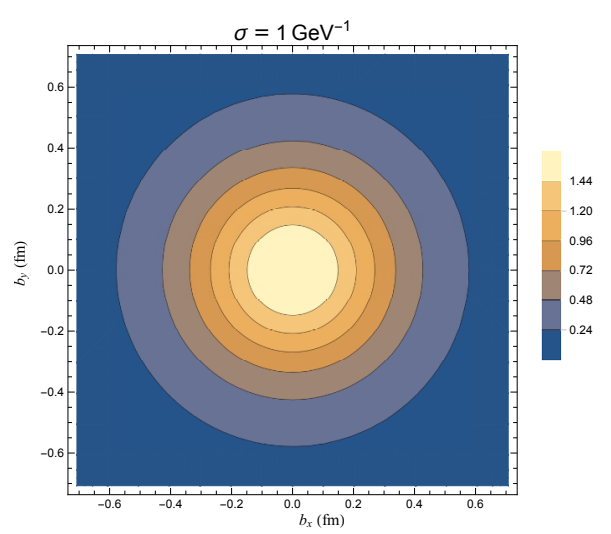

(b) $q_{\sigma}(b)\left(\mathrm{fm}^{-2}\right)$.

Figure 2. Contour plots of the IPD $q(b)$ with a wave packet with $\sigma=1 \mathrm{GeV}^{-1}$. 